\title{
News from the San Antonio Breast Cancer Symposium 2014
}

\author{
Rachel Wuerstlein $^{a} \quad$ Alessandra Gennari ${ }^{b}$ Cornelia Liedtke ${ }^{c}$ Frederik Marmé $^{d}$

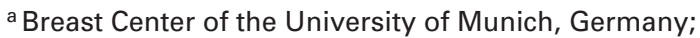 \\ b Galliera Hospital, Genoa, Italy; \\ c Department of Gynecology and Obstetrics, University Hospital Lübeck, Germany; \\ ${ }^{d}$ National Center for Tumor Diseases and University Hospital of Heidelberg, Germany
}

\begin{abstract}
Question 1: There Was a Special Forum Held on the Question 'Accelerating Drug Development, Companion Diagnostics and their Approval'. What Is Your Part in the Field of this Question and Where Do You See Possible Improvements in the Near Future Regarding the Situation in Your Environment?
\end{abstract}

Gennari: We are currently focused on receptor functional imaging to better select metastatic breast cancer patients who really respond to endocrine therapy. I think that implementation of drug directed diagnostics is a key issue for future research and drug approval.

Liedtke: Since I am both a clinician and a translational researcher, I do have a strong opinion with regard to this aspect and do think that it is inevitable that novel targeted agents need to be developed side by side with (efficacy) biomarkers. 2 important goals may be achieved with respect to this: (i) from a clinician's view I acknowledge that patients will be spared unnecessary toxicity since inefficacious agents will not be employed if the biomarker is not present and (ii) from a clinical/translational research view I see an increased potential for novel agents to be established and licensed if they are tested and developed further in a group of patents enriched for chance of response through presence of a biomarker.

Marmé: In order to make optimal use of our rapidly growing comprehensive molecular understanding of the heterogeneity of human breast cancer, new approaches of drug development are needed. As discussed in the special forum this might include the co-development of a combination of two novel agents in parallel and a wider implementation of biomarker driven trials. A compelling scientific rationale for novel-novel combinations is a prerequi- site. In these early days of personalized oncology we learned how much context matters. Whereas vemurafenib works fine in melanoma patients with BRAF V600E mutations, no efficacy is seen colorectal cancers with the same mutation. In vitro screening approaches for synthetic lethality can be successfully used to identify drug combinations.

However, as subsets of patients become smaller and smaller this will require an increased collaborative effort to succeed. In addition, an earlier collaboration between industry and licensing bodies leads to an accelerated assessment and development of innovative drugs.

\section{Question 2: Should All Patients with Primary TNBC Get Platinum-Enriched Regimens? How Do You Advise this Patient Group in the (Neo)Adjuvant Setting?}

Gennari: This issue is still unclear. At present all treatment options should be taken into account; however there is an improved outcome in BRCA mutated patients with platinum salts.

Liedtke: Although data with regard to platinum efficacy in patients with triple negative breast cancer (TNBC) in the curative (i.e. neoadjuvant) setting I strongly advise against broad use of these agents in all patients. This hesitation is particularly based on (i) toxicity concerns associated with combination therapy and (ii) lack of a biomarker allowing patient stratification for/against platinumbased chemotherapy. Although data presented at this year's SABCS suggest increased platinum efficacy for patients with metastatic TNBC harboring a BRCA1 mutation there is yet no such evidence in the curative setting. At our institution we use platinum containing therapy as part of clinical trials. Outside of clinical trials we use

\section{KARGER}

Fax +497614520714

\section{(c) 2015 S. Karger GmbH, Freiburg}

$1661-3791 / 15 / 0101-0061 \$ 39.50 / 0$ 
patient age and response to the first 2-4 cycles of anthracycline based chemotherapy (EC) to stratify patients with regard to platinum chemotherapy. Consequently, patients at young age $(<50$ years) and with limited response to $2-4$ cycles of EC will be offered a carboplatin-taxane combination chemotherapy regimen. Stratification based on age reflects both (i) data from our own group demonstrating a particularly adverse prognosis among patients with TNBC less than 50 years of age and (ii) concerns regarding treatment safety (particularly hematologic toxicity).

Marmé: The data of the TNT trial presented by Andrew Tutt are consistent with data on platinum for triple negative patients in other settings, e.g. the neoadjuvant GeparSixto trial. Platinum should be incorporated early in the treatment of $B R C A$-associated tumors. In $B R C A$-associated tumors the overall response rate was twice as high for carboplatin as directly compared to docetaxel (68 vs. $33 \%$ ) with a positive test for interaction between treatment and BRCA status. This translated into a superior progession free survival. The investigators should not only be congratulated for conducting this overdue academic trial but also on the prospective incorporation of the most important translational questions aiming at the identifying markers to define a group of non- $B R C A$ associated tumors that would equally benefit from platinum and the presentation of this data alongside the clinical data.

Neither the analyses based on the PAM50 intrinsic subtype, the immunohistochemical surrogate subtype definitions nor the Myriad HRD assay (which showed some promising results in a small neoadjuvant trial) were able to predict a 'BRCAness' associated with superior efficacy of platinum. However, this trial also provides evidence that platinum shows clinically meaningful activity even in non-BRCA associated metastatic TNBC and constitutes an additional treatment option with an entirely different toxicity profile.

The most prudent way to incorporate platinum in the treatment in early breast cancer is in the neoadjvuant setting. Thus far we have data from 2 randomized neoadjuvant trials in TNBC (GeparSixto, CALGB40603) that have demonstrated a significant increase in pCR from the addition of carboplatin and one smaller trial, which failed to do so. At least from GeparSixto, we know that this benefit is restricted to $B R C A$-associated tumors or patients with a strong family history. There are currently no survival data and the CTNeoBC pooled analysis has failed to provide an answer to the important question which increment in $\mathrm{pCR}$ rate will translate into a survival benefit. In addition the prognostic role of $\mathrm{pCR}$ in BRCA-associated TNBC is still unclear (Paluch-Shimon et al. ASCO 2014 \#1023). Weighing risks and benefits, platinum can be incorporated in the neoadjuvant treatment of triple negative patients in selected cases based on baseline risk (e.g. stage), $B R C A$ status, response to AC (doxorubicin-cyclophosphamide) or EC in case of sequential regimens. I personally prefer a regimen of dosedense EC, followed by paclitaxel weekly $+/$ - carboplatin (AUC6 $\mathrm{q} 3 \mathrm{w}$ ). Today there is no compelling evidence for the use of platinum in the treatment of early non-BRCA-associated TNBC.

\section{Question 3: What Is Your Take-Home Message from the Session 'Controversies: Contralateral Prophylactic Mastectomy'? From Next Week on, Who Will Get this Option in Your Center?}

Gennari: Contralateral prophylactic mastectomy remains an option in high-risk patients and in patients with particular hereditary make-up. Overall, in Italy conservative surgery will be the first option in most of the patients.

Liedtke: Practice at our center regarding prophylactic contralateral mastectomy (PCM) has not changed since SABCS. We do not routinely suggest PCM to our patients, however, PCM will be performed if patients despite intensive counseling express a strong wish to undergo this procedure. Overall, we do take aspects such as tumor biology and response to therapy (i.e. a pathologic complete response in patients with TNBC or HER2-positive breast cancer) into account.

Marmé: There has been a strong increase of bilateral mastectomies for unilateral breast cancer especially among younger women in the United States. The majority of these patients do not have major genetic or familial risk factors and the decision is mostly made by the patients themselves. Recent studies show that decisions are often based on a misperception of the true risk of contralateral breast cancer and the impact on survival. As doctors are still the most important source of information, it is on us to provide adequate patient centered information and psychosocial support. Ann Partridge stated in her disclosures 'I don't think I have the right answer nor is there likely to be a clear answer in response to the current trend in bilateral mastectomies...' and I agree.

There will not be a change in practice after this year's SABCS in our center. In my perception, this trend has not reached Europe or at least Germany to the same extend observed in the US. However, we need to improve our skills in communicating risks. A patient expressing the wish for bilateral mastectomy for unilateral breast cancer needs to be informed in a way that she is able to correctly perceive and understand the risks of contralateral breast cancer, its implications for reconstruction and for chances of survival. The data from the German Consortium for Hereditary Breast and Ovarian Cancer with regard to mutational status and age at diagnosis are helpful in guiding decisions for $B R C A$-associated as well as sporadic breast cancer with a family risk. One of the biggest challenges is that patients want to take this decision when acutely confronted with the primary diagnosis and to not want to postpone surgery to take their time to make the decision concerning the contralateral breast. It is important to point out that this decision might be more wisely taken if genetic counseling and more time for reflection were granted. Therefore one option is to defer the decision of contralateral prophylactic mastectomy, e.g. during neoadjuvant chemotherapy if this is indicated. If a patient understands her risks and has been well informed and taken her time to make this decision, we should state our opinion but are not in a position to refuse this whish. 
In future, additional risk factors, such as breast density and panels of low- and intermediate-risk genes will further help to counsel patients. But at the present state, they have to be regarded as experimental with unknown implications for clinical management and should not be used in routine clinical practice.

\section{Question 4: We All Got Used to Genome-Directed Therapeutic Management in HR-Positive, HER2- Negative Early Breast Cancer and See Promising Results. Which Trials Did Change Your Procedures Most in the Last Years? Do You Routinely Offer these Tests? How Did Management Patients Change in Your Setting?}

Gennari: We are still waiting for the results of Mindact and TailorX. We do not routinely offer these tests since they are not reimbursed in Italy.

Liedtke: I strongly believe that genomic testing allows for a safe reduction in chemotherapy application frequency among patients with breast cancer and therefore may lead to decreased treatment toxicity. At our institution, we are currently accruing patients to the WSG ADAPT study, in which patients with hormone receptor (HR)-positive breast cancer are assigned to receive chemotherapy or not based on (i) a priori risk assessment through clinical / pathological parameters and genomic testing and (ii) dynamic KI67 measurements during 3 weeks of endocrine therapy. It is estimated that this concept may allow for a reduction regarding chemotherapy use by as much as $60 \%$ without putting patients at increased risk of recurrence. Outside of clinical trials we use genomic testing according to the AGO recommendations, i.e. in cases with borderline prognosis (G2 tumors) or patients with discrepant biologic findings.

Marmé: I personally would be reluctant to call the implementation of any of the current commercially available prognostic multigene tests, to which you allude, a genome-directed therapeutic management. The term genome-directed should remain reserved for decisions based on more comprehensive analysis of the entire genome (such as new generation sequencing based technologies) and not just expression of a limited number or mRNAs measured by RT-PCR.

However, the data from retrospective analyses of prospective trials during the last decade demonstrate that these tests robustly predict prognosis in HR-positive, HER2-negative breast cancer. There is no data comparing the performance of these tests directly and there probably never will. Apart from the studies validating the various multigene assays, the most important studies guiding my practice are the comparison of the Recurrence Score and the IHC4 score on the TransATAC data set as it provides principal insight into a comparison of the prognostic potential of routine IHC markers with such multigene assays and in addition the international Ki67 reproducibility study as it teaches the limitation of Ki-67 testing.
Today we routinely offer these tests to patients with HR-positive, HER2-negative, grade 2 tumors with a Ki67 $>10 \%$ and $<35 \%$ or progesterone receptor $<20 \%$. In addition rarely to grade 1 and grade 3 which display a discrepancy between grade and Ki-67.

\section{Question 5: This Year, There Was a Huge Session of Insights into Metastatic Progression and Prevention and Treatment of Metastasis at SABCS - Commonly More Oriented towards Early BC. What Was the Main Information for You from this Session? Will this Be Practice Changing for You?}

Gennari: Usually we perform aggressive follow up in most of the patients with metastatic breast cancer since overall survival improvements are possible in this patient population with unfavorable prognosis, but only if disease burden is limited and the most active regimens are used.

Liedtke: I do not feel that these insights warrant immediate translation into the clinical practice, however, they do provide a basis for novel scientific hypotheses.

Marmé: We have been presented several important pathways / mechanisms of metastatic progression from preclinical models. There seems to be a genetic program leading to the expression of basal markers (e.g. CK14), which are crucial in leading collective invasion throughout all breast cancer subtypes. Also, LIFR and YAP signaling has been implicated in metastatic progression from preclinical models. All of these markers seem to be prognostic and their inhibition can block metastasis formation in vivo. A first phase II study of FG-3019 targeting a YAP downstream target gene is underway in metastatic pancreatic cancer, showing promising results.

However, one of the barriers for clinical development of agents disrupting mechanisms of metastasis formation is the current concept that novel agents have to be tested in phase I/II trials in the advanced or metastatic setting and demonstrate the ability to shrink tumors. Based on their mode of action, even if they work, they are unlikely to shrink existing tumors. Before any of this can become practice changing, we need a more comprehensive understanding of the underlying processes and how to block them and in addition come up with new clinical development strategies. Further, it remains unclear if disruption of these mechanisms at time of diagnosis is not even too late to ultimately prevent metastasis formation and death of patients.

\section{Question 6: Will the Data Presented at SABC Change Your Recommendation in Endocrine Treatment of Premenopausal BC Patients? What Is Your Standard of Care for Patients $<35$ ?}

Gennari: No, not in the vast majority of premenopausal patients. We routinely use the combination of an LH-RH agonist plus 
tamoxifen. LH-RH plus aromatase inhibitor can now be considered but this has to be balanced against the long-term adverse effects on bone health in a young (and possibly cured) breast cancer patient.

Liedtke: Although data from SOFT / TEXT are encouraging (particularly) with regard to treatment efficacy I find the toxicity result of these analyses presented right after the efficacy presentation quite worrisome (again for the subgroup of patients at particularly young age (i.e. $<35$ years)). Therefore, I would not change my clinical management for all premenopausal patients with HR-positive breast cancer. Instead, I see an important need to develop riskof-recurrence based recommendations within this group of patients which may include age but also other clinical / pathological factors.

Marmé: The data will open a door to ovarian function suppression (OFS) in selected cases again, only 2 years after it has disappeared in this context from our AGO treatment guidelines. However, absolute benefit of any therapy is governed by absolute risk. Low risk will inevitably mean little benefit. Thus far, we don't have any reliable overall survival data in the SOFT and TEXT trials and it is unclear if potential increases in mortality from other causes due to OFS will not make up for the benefit. One of the main messages from the SOFT trial is that doctors are able to identify premenopausal women with HR-positive breast cancer at low risk of recurrence that fare extremely well without chemotherapy and on tamoxifen alone. These results are very reassuring and OFS clearly doesn't play a role in these patients! A meaningful benefit in terms of breast cancer free interval from OFS was seen in patients with prior chemotherapy (reflecting a higher baseline risk) or in the very young ( $<35$ years) patients. However, almost half of the events prevented reflect either local recurrences or contralateral breast cancer. In this age group genetic risk is important and prophylactic salpingo-oophorectomy cuts the risk of $B R C A$-associated of contralateral breast cancer by half. BRCA mutational status is unknown in SOFT.

We should remain reassured that we are able to define a group of patients at low risk even if younger than 35 . Young patients at high risk (stage, biology) need to be informed about the option of OFS, including its detrimental effects on several aspects of quality of life. For high-risk patients OFS plus tamoxifen or exemestane is a treatment option dependent on the tolerability in the individual patient. I would not make this decision solely based on age; patients at high risk (warranting chemotherapy) who are older than 35 might benefit as well.

\section{Question 7: Your Personal View. What Was the Most Relevant Finding at SABCS 2014?}

Gennari: In a conference too much focused on translational (but not applicable to clinical practice) research, the most important news was the demonstration in the GeparSepto trial of the superior activity of nab-paclitaxel as compared to weekly paclitaxel.
Liedtke: Apart from the results of the SOFT / TEXT trials I was pleased to see the results from the German Breast Group (GBG) GeparSepto study which demonstrated superiority of nab-paclitaxel over paclitaxel in the neoadjuvant setting - particularly among patients with TNBC. First, these results are clinically encouraging since they may serve as a basis for optimizing taxane based chemotherapy regimens. Moreover, there are several clinical trials currently ongoing that include nab-paclitaxel as taxane such as the WSG ADAPT study which randomized patients to receive either nab-paclitaxel/carboplatin or nab-paclitaxel/gemcitabine. This trial just recently stopped accrual based on a pre-specified interim analysis. The results of GeparSepto support the choice of nab-paclitaxel as taxane in ADAPT-TN.

Marmé: From a clinical point of view the data most relevant for our daily practice are the TNT as well as the SOFT study. In addition, this year's SABCS made it clear that we have arrived at a new age, beyond gene expression profiling and into a more comprehensive genomic understanding of the diversity of human breast cancers based on next generation sequencing tools. Using these tools we are able to investigate the role in intra-tumor genetic heterogeneity in resistance and treatment failure and can start to find strategies to prevent this. We will have to learn to make use of the technologies and to successfully translate results into clinical practice during the next decade.

Last but not least, immunotherapy has finally demonstrated first exciting results and we can hope, that this will enter clinical practice in the near future. The first phase I clinical trials on immune checkpoint inhibition with anti-PD-1 and anti-PD-L1 antibodies that were presented at San Antonio were restricted to TNBC patients. It will remain our task to identify the right patients for such immune checkpoint blockade strategies in the future.

\section{Participants}

\author{
Alessandra Gennari, MD, PhD \\ Galliera Hospital \\ Mura delle Cappuccine 14, 16128 Genoa, Italy \\ alessandra.gennari@galliera.it \\ PD Dr. med. Cornelia Liedtke \\ Konservative Tumortherapie / Studienzentrale \\ Klinik für Frauenheilkunde und Geburtshilfe \\ Universitätsklinikum Schleswig-Holstein / Campus Lübeck \\ Ratzeburger Allee 160, 23538 Lübeck, Germany \\ cornelia.liedtke@uksh.de \\ PD Dr. med. Frederik Marmé \\ Nationales Centrum für Tumorerkrankungen und \\ Universitätsfrauenklinik Heidelberg \\ Im Neuenheimer Feld 460, 69120 Heidelberg, Germany \\ frederik.marme@med.uni-heidelberg.de
}

